\title{
Design of Pet Robots with Limitations of Lives and Inherited Characteristics
}

\author{
Tomoko Yonezawa \\ Kansai University \\ 2-1-1, Ryozenji, Takatsuki \\ Osaka, Japan \\ yone@kansai-u.ac.jp
}

\author{
Naoto Yoshida \\ Kansai University \\ 2-1-1, Ryozenji, Takatsuki \\ Osaka, Japan \\ k463362@kansai-u.ac.jp
}

\author{
Kento Kuboshima \\ Kansai University \\ 2-1-1, Ryozenji, Takatsuki \\ Osaka, Japan
}

\begin{abstract}
In this paper, we propose a framework of life duration and inheritance for pet robots to make them have original characteristics in their limited lives. The purpose of our research is to develop a pet robot that enables the user to treat the robots as though they had real lives from the viewpoint of importance of life and pleasure of birth through the breeding of robots. The characteristics of bodily motions are inheritable by the next generation. The robots also change their behavior corresponding to elapsed time from birth.
\end{abstract}

\section{Categories and Subject Descriptors}

H.5.2 [Information Interfaces and Presentation (e.g., HCI)]: User Interfaces

\section{General Terms \\ Design}

\section{INTRODUCTION}

In the recent half century, the number of people who keep pets such as dogs or cats grew [1]. The main reasons to keep pets are anticipated improvement to the mental, social, and physiologic health statuses of humans $[2 ; 3$, etc], or to the family relationship [4], according to each owner's image[5]. The importance of companion animals are known by the roles of them as members in families[6], therapeutic companions in animal therapy [7], or social/developmentalmind education of children[8]. However, at the same time, companion animals bring the problems of breeding, disposal of excreta, and feeding.

On the other hand, there have been pet robots that were developed based on breakthroughs in robot engineering and psychological and social interaction. These pet robots also bring similar effects of healing to animal therapy through emotional interaction $[9 ;, 10$, etc. $]$. Thus, the effectiveness of the artificial presence is shown in various scenes; however, the expressions of robots and interactions between human and robot are limited, with few variations, so that users get bored using them as daily companions. The greatest difference between animal companions and pet robots is the way in which their lives are limited.Our lives are limited, and the lives of their animal companions are limited. As such, the communications between them are limited, precious experiences. We considered that humans instinctively understand the preciousness of the living companion and that the affective emotions are developed through the interactions.

In this research, we introduce a pet-robot framework with the concept of limited duration of life and characteristic inheritance for further possibility of user's affection. Growth, aging, and death correspond to duration of life and elicit the feeling of precious communication. The inheritance of motion characteristics is adopted for affective interaction through multiple generations. In this paper, we discuss the expressions of life duration and inherited characters for the living-being-like presences.

\section{RELATED RESEARCHES}

There are several researches on interaction and affection. Our focus is on the improvement of affective communication between human and robot. First, we focus on the relationship of human and companion animals. The end of the life of a pet gives us a strong shock known as pet-Loss [11], that sometimes develops into a large problem of mental health even pets normally improve our mental states. However, these strong hookups between pets and humans grow the strong emotions of affection and confidence with precious memories.

Robots have characteristics of both artificial machines and animal-like presences. The latter impression is enhanced by the growth of the characters as seen in AIBO [12], the smooth and natural motions like animals as seen in PLEO $[13$, etc.], and the interactivity as discussed in various communication robots $[14 ;, 15$, etc. $]$ Still the prepared and limited interaction cannot develop into the sense that they are real, living beings. In this paper, we develop the possibility of the communication robot by both the limitation of life duration with growth and aging and the inheritance of characteristics.

Affective emotion is strongly created through intimate communication. Such communication provides us with a feeling of a living being for a partner [16]. Meng et al. introduced an involuntary expression on the robot's surface as though it were a living being with skin [17]. Thus, uncontrollable ex- 


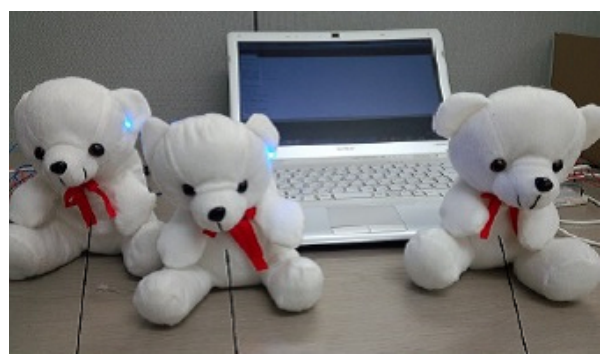

Figure 1: System views of birth and inheritance between parents and child robot

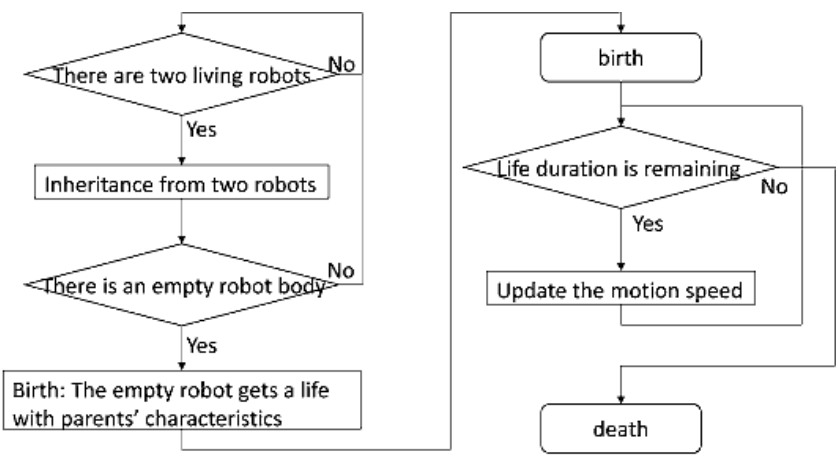

Figure 2: System flow

pressions are expected to be perceived as outlets of a physical state with true internal feelings. In this research, we focus on duration of life and inheritance as biological expressions. The long term communication between human and robot reflects the modal change of these expressions.

\section{ROBOT WITH LIMITED LIFE AND IN- HERITED CHARACTERISTICS \\ 3.1 System Structure}

Our proposed system is realized by a pet robot covered with bear-like stuffed toys. Two servomotors and a pressure sensor were enclosed in its head for two degrees of freedom: vertical and horizontal. An AVR (Arduino UNO) controls the reactive motion of the robot and captures the sensor value. A full-color LED is attached to the ear of each pet robot. Each robot is connected to a PC that controls the events of inheritance, birth, aging, and death through serial connections in the current implementation.

In order to implement the inherited characteristics, the conceptual model of the birth is prepared as follows. When there are two living-state robots with an extra robot that is not alive, a new characteristic that is organized by two parents' robots is adopted for the extra robot. The organized characteristic is realized in the extra robot; that is a conceptual birth. Figure 1 shows the situation of the inheritance and the birth.

In this system, the concept of limited life treats the birth, the death, growth/aging, and the concept of characteristic inheritance to treat the motion of the head. Figure 2 shows the system flow with our proposed two features: limitation of life and inheritance for interactive robots. As a tentative no life

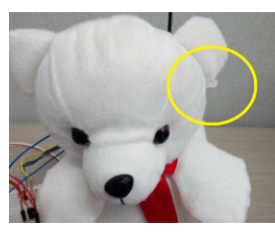

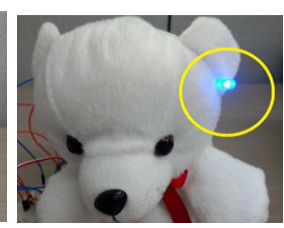

old aged

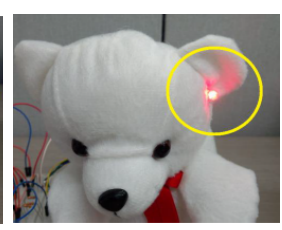

young
Figure 3: LED color changed by rest of life duration

Table 1: Prepared characteristics of motion

\begin{tabular}{l|l}
\hline Characteristic & Motion of head \\
\hline cheer(A) & look upward, move left and right \\
live-wired & move left and right swiftly \\
pessimistic & look at the ground, move slowly \\
laid-back & move slowly toward up, down, left and right \\
\hline
\end{tabular}

design, life duration for each individual has a fixed duration, such as one year. The duration could become variable as one of inherited characteristics.

\subsection{Expressions for Limited Life}

We consider that the expressions for aging or growth, the rest of the duration of life, or the situation of life, change in order to make the owner assume that there is a limit and end to life. In our system, we prepared two types of expressions for the remainder of life, as follows.

1. A color LED displaying the elapsed time from blue to red as shown in Figure 3: The color LED expression is very typical for mechanical expression; however, it is easy to observe without long-term interaction.

2. Change of characteristics in motions and reactions: The speed of motion or reaction is different between that of children or elderly people as seen in human or animal lives, so it is considered that the expression with motions is more familiar to our feelings.

\subsection{Inherited Characteristics}

Inheritable characteristics are shown in Table 1. These four types are prepared as in Russell's emotional circumplex model [18]. The algorithm of the inheritance is based on Mendel's law. The order of dominance is (A), (B), (C), to (D). When the parents' genes are $(\mathrm{AB})$ and $(\mathrm{CD})$, the children could become $(\mathrm{AC}),(\mathrm{AD}),(\mathrm{BC})$, or $(\mathrm{BD})$. The dominant inheritance shows only (A) or (B) characteristics in next generation.

\section{PRELIMINARY EXPERIMENTS FOR LIFE AND AFFECTION}

Purpose of the experiment: We aimed to verify 1) whether the limited duration of a robot's life causes the user's affective emotion, and 2) which expression of the remaining duration of life is appropriate.

Hypotheses: I) The limitation of life elevates the affective emotion of the user. II) The lifelike expression of the rest of life duration elevates the affective emotion of the user.

Participants: 20 university students aged from 19 to 22 years old (11 males and 9 females.) 
Conditions: We prepared four conditions according to two factors: 1) with or without limitation of life duration, and 2) two different expressions of the rest of life duration. The conditions are as follows:

A) No limitation of life duration: An LED color expresses the remainder of life duration. This condition did not show any motion of the robot.

B) With limitation of life duration: An LED color expresses the remainder of life duration. This condition did not make any motion, but LED color was changed over time.

C) No limitation of life duration: The robot's motion expresses the remainder of life duration. This condition showed the robot's motion that was not changed over time.

D With limitation of life duration: The robot's motion expresses the remainder of life duration. This condition showed as gradually changed motion over the passage of time.

Procedures: The participants were instructed to observe the robot and its behaviors during a one-minute session for each condition. After each session, the participants evaluated the impression for the statements of evaluation items, as follows.

Evaluation items: The participants made the evaluation using a five-point scale rating of the relevance (5: very relevant, 4: somewhat relevant, 3: even, 2: somewhat irrelevant, 1: irrelevant) of the following statements, except Q16, which was a free description.

Q1 You were eager to know about the robot.

Q2 The robot seemed interesting.

Q3 You wanted to interact with the robot.

Q4 You felt discomfort.

Q5 The robot became cheerier.

Q6 The robot seemed to decline.

Q7 The robot gradually changed its age.

Q8 You began to like the robot.

Q9 You did not want to become involved with the robot.

Q10 You wanted to be with the robot much longer.

Q11 You thought the robot was broken in the session.

Q12 You felt a sad impression about the robot.

Q13 The robot seemed to live.

Q14 You became interested in the robot.

Q15 You wanted to communicate with the robot much longer

Q16 (free description) Please describe the change in the

robot and the meaning of it as you felt.

Q17 You were delighted.

Q18 You became sad.

Q19 You became irritated.

Q20 You felt nothing.

Results: Free descriptions for Q16 showed the possibility that there were several participants who considered the change of expression as a change of the robot's emotion.

Figure 4 shows the results of MOS (mean opinion score), and Table 2 shows the results for ANOVA (analysis of variance).

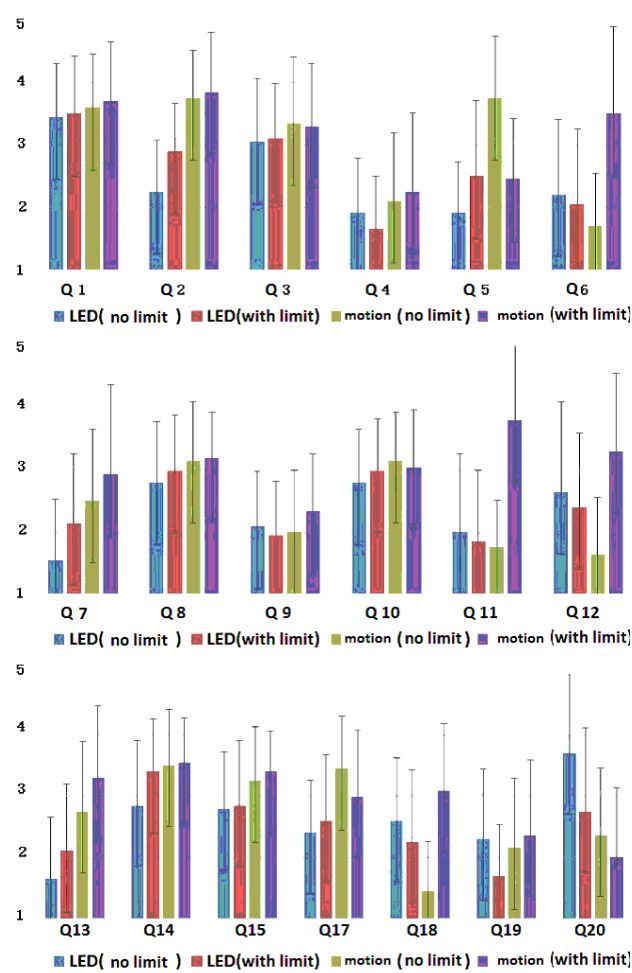

Figure 4: MOS results

Q2, Q7, Q11, Q13, and Q20 showed significance for both: limitation and expression factors while Q1, Q3, Q8, Q9, Q10, and Q19 did not show any significance.

There are also significant differences by each factor. Q5, Q15, and Q17 showed significance of only the expression factor. Q6, Q12, and Q18 showed significances only for the limitation factor. There were simple main effects in Q5, Q6, Q11, Q12, and Q18. Thus, both hypotheses I) and II) were confirmed.

\section{DISCUSSION}

As shown in the significant results of the expression factor, the expression of motion was preferred than LED display for the remainder of life duration. The results show the possibility of the advantage of lifelike expression; however, there is another possibility that the motion is stronger than the LED signal, so the participants were interested in the stimr.uli. Accordingly, we should verify the combination of them in order to separate the enclosed factors in the expression factor.

The results for the limitation factor showed the significance for interest while it did not show any significant difference for affective emotion contrary to our expectation. It is considered that the pet robot in this experiment did not provide interactivity during the session, so the user's experience and memory did not develop a precious feeling for the interaction.

Several results with significances for both factors showed simple effects, and the interaction effects were confirmed. 
Table 2: ANOVA results

\begin{tabular}{|c|c|c|c|c|}
\hline \multirow{2}{*}{$\begin{array}{l}\text { factor } \\
\text { item }\end{array}$} & \multicolumn{2}{|c|}{ expression } & \multicolumn{2}{|c|}{ limitation } \\
\hline & $\mathrm{F}_{(19)}$ & $\mathrm{p}$ & $\mathrm{F}_{(19)}$ & $\mathrm{p}$ \\
\hline Q1 & 0.921 & 0.349 & 0.263 & 0.614 \\
\hline Q2 & 48.58 & $<.01^{*}$ & 5.98 & $0.024^{*}$ \\
\hline Q3 & 1.462 & 0.242 & $<0.01$ & $>0.99$ \\
\hline Q4 & 4.252 & $0.0532+$ & 0.083 & 0.776 \\
\hline Q5 & 14.45 & $0.0012^{*}$ & 5.783 & 0.027 \\
\hline Q6 & 4.344 & $0.0509+$ & 8.442 & $<.01^{*}$ \\
\hline Q7 & 17.18 & $<.01^{*}$ & 6.655 & $0.018^{*}$ \\
\hline Q8 & 1.420 & 0.248 & 0.629 & 0.438 \\
\hline Q9 & 0.426 & 0.522 & 0.309 & 0.585 \\
\hline Q10 & 1.152 & 0.297 & 0.073 & 0.789 \\
\hline Q11 & 9.788 & $<.01^{*}$ & 13.0 & $<.01^{*}$ \\
\hline Q12 & 0.033 & 0.857 & 4.811 & $0.041^{*}$ \\
\hline Q13 & 45.48 & $<.01^{*}$ & 12.67 & $<.01^{*}$ \\
\hline Q14 & 4.406 & 0.049 & 4.171 & $0.0553+$ \\
\hline Q15 & 8.261 & $<.01^{*}$ & 0.458 & 0.507 \\
\hline Q17 & 8.982 & $<.01^{*}$ & 0.253 & 0.621 \\
\hline Q18 & 0.449 & 0.511 & 6.676 & $0.0182^{*}$ \\
\hline Q19 & 0.087 & 0.362 & 1.974 & 0.1762 \\
\hline Q20 & 23.3 & $<.01^{*}$ & 9.148 & $<.01^{*}$ \\
\hline \multicolumn{5}{|c|}{$\mathrm{p}<.05:{ }^{*}<.10:+$} \\
\hline & \multicolumn{3}{|c|}{ (simple effects) } & \\
\hline & Q5 & \multicolumn{3}{|c|}{$\mathrm{A}(\mathrm{b} 1), \mathrm{B}(\mathrm{a} 1), \mathrm{B}(\mathrm{a} 2)$} \\
\hline & Q6 & \multicolumn{3}{|c|}{$\mathrm{A}(\mathrm{b} 2), \mathrm{B}(\mathrm{a} 2)$} \\
\hline & Q11 & \multicolumn{3}{|l|}{$\mathrm{A}(\mathrm{b} 2), \mathrm{B}(\mathrm{a} 2)$} \\
\hline & Q12 & \multicolumn{3}{|c|}{$\mathrm{A}(\mathrm{b} 1), \mathrm{A}(\mathrm{b} 2), \mathrm{B}(\mathrm{a} 2)$} \\
\hline & Q18 & \multicolumn{3}{|c|}{$\mathrm{A}(\mathrm{b} 1), \mathrm{A}(\mathrm{b} 2), \mathrm{B}(\mathrm{a} 2)$} \\
\hline
\end{tabular}

From the results, it is conjectured that the combination of both limitation of life duration and the lifelike expression of the remainder of life duration with the robot's motion, enables drawing the user's interest with a possibility of affective emotion. In addition to a preliminary test in quick evaluations, we need to verify further the effectiveness of using our proposed system in long-term interactions.

\section{CONCLUSION}

This paper introduced our new concept of living-being-like pet robots within a framework of life duration and inheritance of original characteristics. From the viewpoint of importance of precious life and birth through interaction with the robots, our goal was set to enable the user to treat the robots as though they had real lives. Corresponding to the elapsed time, the robot's behaviors are changed from birth to death. The characteristics of bodily motions of the parents' robots are inherited by the child generation. From the results of the evaluations, we confirm the possibility of lifelike expressions of the remainder of life duration to draw the affective emotion of the user while the limitation of life duration simply provides the user's interest. In the future, we need to verify the effectiveness of the inheritance; that is, whether the child robot can take over the affection of the user to the parents' robots, while involving multiple interactions among the parents' robots, child robot, and human user.

\section{Acknowledgment}

This research was supported in part by JSPS KAKENHI $15 \mathrm{H} 01698$ and 25700021.

\section{REFERENCES}

[1] C. N. Carter, "Pet population control: Another decade without solutions?." Journal of the American Veterinary Medical Association, 1990.

[2] Barrie Gunter. "Pets and people: The psychology of pet ownership." Whurr Publishers, 1999.

[3] Jean E. Veevers, "The social meaning of pets: Alternative roles for companion animals." Marriage \& Family Review 8.3-4 : 11-30, 1985.

[4] Froma Walsh. "Human-Animal Bonds II: The Role of Pets in Family Systems and Family Therapy." Family process 48.4, 481-499, 2009.

[5] Russell W. Belk, "Metaphoric relationships with pets." Society \& Animals 4.2, pp.121-145, 1996.

[6] Ann Ottney Cain. "Pets as family members." Marriage \& Family Review 8.3-4, pp. 5-10, 1985.

[7] Judith Gammonley and Judy Yates. "Pet projects: animal assisted therapy in nursing homes." Journal of Gerontological Nursing, 17(1): 12-5, 1991.

[8] Gail F. Melson, "Child development and the human-companion animal bond." American Behavioral Scientist 47.1, pp.31-39, 2003.

[9] T. Shibata, T. Mitsui, K. Wada, A. Touda, T. Kumasaka, K. Tagami, and K. Tanie. "Mental commit robot and its application to therapy of children." Proc. of IEEE/ASME Conf. on. Advanced Intelligent Mechatronics, Vol. 2. 1053-1058, 2001.

[10] Masahiro Fujita. "On activating human communications with pet-type robot AIBO." Proceedings of the IEEE 92.11: 1804-1813, 2004.

[11] Bruce S. Sharkin, and Audrey S. Bahrick. "Pet loss: Implications for counselors." Journal of Counseling \& Development 68.3, pp. 306-308, 1990.

[12] M. Fujita, "AIBO: Toward the era of digital creatures," Int. J. Robot. Res., vol. 20, no. 10, pp. 781-794, 2001.

[13] A. Curtis, J. Shim, E. Gargas, A. Srinivasan, and A. M. Howard. "Dance dance Pleo: developing a low-cost learning robotic dance therapy aid." Proc. of Int. Conf. on Interaction Design and Children. ACM, 149-152, 2011.

[14] Cynthia Breazeal. "Toward sociable robots." Robotics and autonomous systems 42.3, pp.167-175, 2003.

[15] Tetsuya Ogata, and Shigeki Sugano. "Emotional communication robot: WAMOEBA-2R emotion model and evaluation experiments." Proceedings of the International Conference on Humanoid Robots. 2000.

[16] Author's paper in Japanese to appear;

[17] Meng Xiaoshun, Naoto Yoshida, and Tomoko Yonezawa, "Evaluations of Involuntary Crossmodal Expressions on the Skin of a Communication Robot," URAI2015, to appear.

[18] James A. Russell, "A circumplex model of affect." Journal of personality and social psychology 39.6, pp.1161-1178, 1980. 